\title{
Double Burden, Non-Communicable Diseases And Risk Factors Evaluation In Sub-Saharan Africa: The Nigerian Experience.
}

\author{
Ekpenyong, C. E ${ }^{1}$, Udokang, N.E ${ }^{1}$, Akpan, E.E, Samson, T.K ${ }^{2}$
}

\begin{abstract}
This study was aimed to assess the age and sex specific burden and associated risk factors of NCDs in adult population of South-South Nigeria. It was a cross-sectional study conducted in Uyo Metropolis, in 2009/2010; with 2780 participants (1447 males and 1333 females) aged 18-60 years. Instruments of survey were: a semi-structured questionnaire, anthropometric and non anthropometric measures using standard procedures. The overall prevalence of NCDs was $32.8 \%$. Disease specific prevalence was as follows: 25\%,14.4\%, 12.7\%, 20.1\% and 10\% for obesity, hypertension, diabetes mellitus, musculoskeletal disorders and respiratory disorders respectively. Males' vs females' prevalence were: $20.7 \%$ vs $29.5 \% ; 12.6 \%$ vs $12.2 \% ; 9.7 \%$ vs $16.0 \% ; 14.0 \%$ vs $26.5 \%$ and $8.6 \%$ vs $7.6 \%$ for obesity, hypertension, diabetes mellitus, musculoskeletal disorders and respiratory disorders respectively. Risk factors with increase odds for NCDs were: age, area of residence, work stress, triglyceride levels and positive family history. Physical inactivity, high total cholesterol level, high general adiposity, high central adiposity and poor dietary habits were equally significantly associated. The high prevalence of NCDs in Nigeria was precipitated by modifiable and un-modifiable life style factors. Intervention programmes should focus on these factors to reverse the trend.
\end{abstract}

Key words: Non-communicable disease, burden, risk factors, adult, Nigeria.

${ }^{\mathbf{1}}$ Department of Physiology, College of Health Sciences, University of Uyo, Akwa Ibom State, Nigeria.

${ }^{2}$ Department of Mathematics, Statistics and Computer Sciences, University of Calabar, Calabar Nigeria.

Corresponding Author Dr. Ekpenyong, C. E : Tel: $+2348023347719,+23480675448487$

E-mail: chrisvon200@yahoo.com 


\section{Introduction}

Non-communicable diseases (NCDs) are increasingly becoming the leading causes of morbidity and mortality worldwide. They encompass a cluster of illnesses which include: Diabetes mellitus, Cancers, Chronic respiratory diseases, cardiovascular disease and musculoskeletal disorders. They are now seen to affect the poor of the poorest countries in the world. The impact is greatest on the poor countries of sub-Saharan Africa of which Nigeria occupy a significant position.

This is because they are often unable to access the education and services required to prevent and treat NCDs. The little health resources remained focused on reducing the already overwhelmed burden of communicable diseases and preventable causes of infant and maternal mortality. Thus, it is not exaggeration to describe the situation in developing countries as an impending disaster; a disaster for health, for society and most of all for national economies. This shift towards NCDs in developing countries dispelled the popular myth that NCDs afflict mostly the affluence (high income) population. However, the developed countries are equally sharing in the scourge, but while the developing countries are facing a double burden, the developed and the high income countries are experiencing a shift in the health trend from communicable to non-communicable diseases.

Non-communicable diseases are the number one world's killer, causing $60 \%$ of all deaths globally (HCC. NCD Alliance 2011), and a staggering 35 million people die every year from these silence killers, of which 18 million are women. In 2008 , this figure rose to 36.1 million (i.e. about $63 \%$ of global deaths), and nearly $80 \%$ of those NCD deaths-equivalent of 29 million people occurred in low and middle income countries with the projection of about 52 million deaths annually by 2030 (WHO, 2011). Also, NCDs will be responsible for three times as many disability adjusted life years (WHO, 2004), and nearly 5 times as many deaths as communicable diseases, maternal, perinatal, and nutritional conditions combined (WHO, 2008). About one-fourth of the global NCDs related deaths take place before the age of 60 years (WHO, 2010). These clusters of diseases represent the biggest threat to global health care and economy, especially at it concerns women (HCC-NCD Alliance, 2011), since prior studies have shown a higher prevalence of some of the predisposing factors in women than men (Khuwaja et al. 2010).

Chronic non-communicable diseases are noted to deliver a two-punch blow to development. They cause billion of dollar in losses of national income, and they push million people below the poverty line (WHO, 2011). It is projected that in the next 10 years, China, India and Britain will lose 558, 237, and 33 billion dollars respectively due to heart disease, stroke and diabetes mellitus (Daar et al. 2007;WHO, 2009). 
In United States 750 billion dollars is spent annually on cardiovascular disease and diabetes mellitus (Venkat Narayan et al. 2010) and about $87 \%$ of all deaths in US are due to NCDs (WHO, 2011). Globally, cardiovascular disease account for most death (about 17 million people annually) followed by cancer (7.6 million), respiratory diseases (4.2 million) and diabetes (1.3 million). These cluster of diseases account for $80 \%$ of disease related deaths (Shukla, 2011).

In Africa, most countries have not conducted risk factor surveys to establish the national based line prevalence rates and accurately quantify the magnitude of the problem. Data from health management information systems is used to estimate the national prevalence status (Mufunda et al. 2006). However distribution of the leading global NCD deaths by the WHO region 2008 showed that cardiovascular disease account for most deaths (1.3 million), followed by cancers ( 0.4 million), respiratory disorders ( 0.3 million), diabetes mellitus (0.2 million) and chronic obstructive pulmonary disorders (0.1 million). This figure is a tip of the iceberg as data from most countries are not available.

In Nigeria, the impact of NCD is enormous and glaring. About 5 million Nigerian may die of NCDs by the year 2015, and diabetes alone is projected to cause about $52 \%$ of the mortality by 2015 . Also, the economic cost of NCDs in Nigeria in 2005 was about 400 million dollars from premature death due to NCDs. By 2015, it is estimated to rise to about 8 billion dollars (HERFON, 2011). At present, about 8 million Nigerians suffer from hypertension and 4 million has diabetes; 100, 000 new cases of cancers are diagnosed each year in Nigeria. Ten thousand Nigerian die annually from injuries from road traffic accident (Chukwu, 2011). These great losses are not just at an individual's levels, but also profoundly affect the family and a country's work force and for the million struggling with poverty, a vicious cycle ensues. Researchers have empirically identified the link between NCDs and globalization, urbanization, demographics, life style transition, socio-cultural factors, poverty, poor maternal, foetal and infant nutrition (Venkat Narayan et al. 2010).

The four major NCDs featuring prominently at the scene include: cardiovascular disease, cancers, chronic respiratory diseases and diabetes mellitus. Also the four main behavioral risk factors include; Tobacco use, physical inactivity, alcohol and unhealthy diet (WHO, 2011).

Almost 6 million people die from tobacco each year, both from direct tobacco and second hand smoke. By 2020, this number will increase to 7.5 million (Mathew et al. 2006). Smoking is estimated to cause about $71 \%$ of lungs cancer, $42 \%$ chronic respiratory disorder and nearly $10 \%$ of cardiovascular disease (WHO, 2011). Also, about 3.2 million people died each year due to insufficient physical activity. People who do not take enough exercise have $20-30 \%$ increase risk of dying prematurely. Regular exercise reduces the risk of cardiovascular 
diseases including high blood pressure, and of diabetes, breast and colon cancers and depression. Insufficient physical activity is highest in high-income countries, but very high levels are now also seen in some middle-income countries especially among women (WHO, 2011). Alcohol intake contributed about 2.5 million deaths annually (about $3.8 \%$ ) of all death in the world.

Similarly, poor dietary habit/obesity contribute about 2.8 million death each year, risk of heart diseases, strokes and diabetes increase steadily with increasing body mass index (BMI) (WHO, 2011). Eight percent of all heart disease, stroke, and type 2 diabetes mellitus and $40 \%$ of all cancers can be prevented (Anderson et al. 2009). Immediate and aggressive action/attention is needed to halt the trend. More effort should be directed towards identifying the major risk factor which may vary in order of significance from country to country and even within country the variation may exist from state to state depending on the sociodemographics characteristics and cultures as it affect lifestyle of a given population. Population measurement of risk factors can be used to describe the distribution of future diseases and knowledge of risk factors can then be applied to shift population distribution of these risk factors.

Thus the aim of this study was to determine the age and sex specific prevalence and associated risk factors for non-communicable diseases in adult population in south-south Nigeria which hitherto has not been documented. We hope the result of this work will help unfold the required interventions necessary to reduce incidence and hence morbidity and mortality associated with NCDs in Nigeria and similar countries all over the world.

\section{SUBJECTS AND METHODS}

\section{Study Design and Population}

This was a cross-sectional multicenter health survey conducted in 2009/2010 within Uyo metropolis South-South Nigeria, in a two primary health care promotion programme after a pre-survey lecture on the menace of Obesity and other associated non-communicable disease in Nigeria delivered by the research coordinator.

A total of 2866 participants were initially recruited for the study, seventy-eight $(2.7 \%)$ were dropped on account of inappropriate age, decline participation, presence of deformity, pregnancy and improper completion of questionnaire. Of the two thousand seven hundred and eighty who met the inclusion criteria, 1447 were males and 1333 were females, aged 18-60 years. Informed consent was obtained from participants and study protocol was approved by the institutional health and ethics board. 


\section{Data Collection}

A semi-structured pretested questionnaire made up of sixty-five items designed to determine the prevalence and risk factors for non-communicable diseases were administered for participants to fill out. For the purpose of this survey, non-communicable diseases were defined as cluster of illnesses which include: diabetes mellitus, hypertension and other cardiovascular diseases, chronic respiratory diseases, musculoskeletal disorders, obesity and cancers. The questionnaire was divided in to two sections: A and B. Section A sought information on socio demographic variables of participants such as; age, sex, marital status, family history of any of the NCDs, educational level, monthly income, employment status, nature of work, work environment, area of residence and type of family. Section B sought information on individual's behavioral, physical and psychosocial risk factors for non-communicable diseases such as physical activity status, dietary habits, smoking or tobacco use, and alcohol intake. Others include biomechanical exposure at work place, (e.g. awkward postures and work pace), and psychosocial work environment (monotonous work, job control, satisfaction, workload and time pressure).

Participants were asked whether they had heart diseases, diabetes mellitus, chronic respiratory disorder and hypertension. Previous history of stroke and regular medication were also elicited. To asses physical activity status, questions were asked based on the 2010 US healthy people physical activity guidelines standard, which recommended 150 minutes of moderate to severe intensity of aerobic physical activity per week in bouts of 10 minutes or more for adults ages between 18 and 64 years. Based on this, participants were classified into two groups; physically active and physically inactive. Dietary habits was assessed by assessing intake of various macronutrients (fat, carbohydrate and protein) vegetables, snacks, sweet drinks, portion size and restaurant/fast food consumption for a period of one year prior to the study. High to very high intake of macronutrients, full portion size consumption, high frequency of fast food/restaurant patronage were all regarded as poor dietary habit, while balanced diet taken 2-3 times a day with a lot of vegetables and fruits in between was regarded as good dietary habits. To asses smoking status, participants were asked whether they had formally smoked, currently smoke, or never smoked. Former smokers were asked whether they had quit less than a year prior to the study period. Those who said they currently smoked at the day of the study were defined as current smokers. Thus they were classified into three groups namely; never smoked, current smokers and ex-smokers. Participants were also classified based on their alcohol intake into two groups namely; regular/current drinkers 
and non-drinkers. Regular drinkers were defined as those who drink for $\geq 10$ days a month for the past six months.

The participant's anthropometric variables (weight, height and waist circumference (WC)) were measured using the standard protocols as approved by WHO, (2003. Body mass index (BMI) was calculated as weight $(\mathrm{kg}) /$ height $\left(\mathrm{m}^{2}\right)$. Overweight was defined as BMI of 25.0-29.9 and Obesity as BMI $\geq 30.0$. Men and women with WC values of $\leq 94$ and $80 \mathrm{~cm}$ respectively, were considered to have a normal WC, whereas men and women with $\mathrm{WC}$ values $>94$ and $>80 \mathrm{~cm}$, respectively were considered to have high WC (obesity). According to WHO and NIH cut offs (WHO, 2008; NIH, 1998).

For the assessment of participants' diabetic status, blood sugar measures were performed using a pre-tested fine test blood glucose monitoring system (Glucometer by Infopia Co., Ltd, Kora). Fasting blood sugar (FBS) test was conducted for those who appeared for the test after overnight fast (at least 8hrs of not eating food). Casual plasma glucose test was conducted for those who had eaten breakfast before appearing for the test, while 2hours post prandial (2hpp) glucose was conducted for all participants. FBS was preferred because of its convenience in a clinical setting and low cost. Casual blood glucose estimation is defined as estimation at any time of the day without regard to the time since last meal, two hours postprandial glucose (2hpp) is defined as two hours since last meal. Diabetes mellitus was diagnosed based on the 2011 Revised Criteria by the Expert Committee on the diagnosis and classification of diabetes mellitus which recommends the diagnosis of diabetes base on:

1. Two fasting Plasma Glucose (2FPG) levels of $126 \mathrm{mg} / \mathrm{dl}(7.0 \mathrm{mmol} . \mathrm{l})$ or higher.

\section{OR}

2. Two, 2hours Post prandial Glucose (2hPPG) reading of 200mg/1 $(11.1 \mathrm{mmol} / \mathrm{L})$ or higher after a glucose load of $75 \mathrm{~g}$

\section{OR}

3. Two casual glucose readings of $200 \mathrm{mg} / \mathrm{dl}(11.1 \mathrm{mmol} / \mathrm{L})$ or higher

OR

4. Glycosylated Hemoglobin of $\geq 6.4 \%$

Participant's blood pressure levels were measured in $\mathrm{mmHg}$ in a sitting position and after a rest of 5 minutes using a pre-tested Accoson sphygmomanometer. This was repeated twice and the average value was used to group participants into hypertensive and non-hypertensive. Hypertension was defined according to the joint National Committee on Prevention, Detection, 
Evaluation and Treatment of High Blood Pressure (Chobanian, 2003) criteria as average blood pressure of $\geq 140 / 90 \mathrm{mmHg}$ after two readings.

For assessment of musculoskeletal disorders, participants were classified into two groups; those with and those without musculoskeletal disorder. Musculoskeletal disorders were defined as disorders affecting muscles, tendons, ligaments and bones.

For chronic respiratory disorders (CRD) participant were classified into two groups: those with and those without chronic respiratory disorders. Chronic respiratory are defined as: chronic diseases of the air ways and other parts of the lungs e.g. asthma, chronic obstructive pulmonary diseases (COPD), lung cancer, cystic fibrosis and occupational lung diseases.

\section{Statistical Analysis}

Data obtained were analyzed descriptively using frequencies and simple percentages. Relationship between each socio-demographic variable and reported cases of each component of non-communicable disease (obesity, hypertension, diabetes mellitus, musculoskeletal disorders and respiratory disorders) and sex was tested using chi-square. Moreover, univariate analysis of the association between non-communicable diseases and each of its possible risk factors was also investigated with the use of chi-square statistical test.

Furthermore, multivariate analysis using multiple logistic regression model which incorporated all possible risk factors and while adjusting for the effect of possible confounders such as employment status, marital status, ethnicity and annual income was also applied. This analysis was done separately for men and women. Based on the results of multiple logistic regression models, odd ratios and its corresponding 95\% confidence interval were estimated. Statistical computations were enhanced with the Statistical Package for Social Sciences (SPSS 17.0). P-values less than 0.05 were considered to be statistically significant.

\section{Results}

Of the 2,780 participants, 1447 (52.1\%) were males and $1333(47.9 \%)$ were females. The distribution of all socio-demographic variables differ significantly between males and females with $\mathrm{P}$ values $=0.000$ obtained for all sociodemographic variables except education whose P-value $=0.008$, Table 1 .

The overall prevalence of non communicable diseases (all diseases cluster) was $32.8 \%$ (912 of 2.780 subjects) with all the risk factors (based on the univariate analysis using chi-square) having a significant relationship with NCDs $(\mathrm{P}<0.05)$, Table 2. 
Prevalence of obesity, hypertension, diabetes mellitus, musculoskeletal disorders and respiratory disorders were as follows; 25\%, 14.4\%, 12.7\%, 20.1\% and $10 \%$ respectively. Males vs females prevalence were as follows; $20.7 \%$ vs $29.7 \%, 12.6 \%$ vs $12.2 \%, 9.7 \%$ vs $16.0 \%, 14.0 \%$ vs $26.5 \%$ and $8.6 \%$ vs $7.6 \%$ respectively. Obesity, diabetes mellitus and musculoskeletal disorders were more prevalent in women while hypertension and respiratory disorders were more in men. Highest prevalence was recorded among participants within the age brackets 34-64, Table 3. The result of incidence of NCDs across age group was also illustrated with cluster bar chart with error bar (see figure 1).

Results of the association between risk factors and prevalence of NCDs in males vs females using multiple logistic regression analysis and depicted by the adjusted odds ratios and 95\% confidence intervals were as follows: age (OR = 4.75, C.I $=3.23-10.60)$ vs $(\mathrm{OR}=5.02$, C.I $=4.83-12.74)$, physical inactivity $(\mathrm{OR}=$ 2.08, C.I $=1.595-2.722)$ vs $(\mathrm{OR}=3.04$, C.I $=2.271-4.062)$, poor dietary habits $(\mathrm{OR}=4.23$, C.I=1.641-10.906) vs $(\mathrm{OR}=4.96, \mathrm{C} . \mathrm{I}=1.893-12.988)$, obesity (OR $=2.7$, C.I $=1.856-4.094)$ vs $(3.72$, C.I $=2.525-5.470)$, work stress $(\mathrm{OR}=2.81$, C.I $=1.899-4.163)$ vs $(\mathrm{OR}=5.23$, C.I $=3.332-8.214)$, area of residence $(\mathrm{OR}=1.53$, C $. I=1.162-2.021)$ vs $(\mathrm{OR}=1.851$, C.I $=1.285-3.084)$, positive family history (OR $=4.18$, C.I $=3.51-4.970)$ vs $(\mathrm{OR}=4.16$, C.I=3.455-5.019), total cholesterol $(\mathrm{OR}$ $=1.51$, C.I=1.198-1.899) vs $(\mathrm{OR}=2.17$, C.I=1.475-3.184).

Results obtained for smoking habits, alcohol consumption, waist circumference (only in males), triglyceride (TG), low density lipoprotein (LDL) and high density lipoproteins (HDL) were statistically insignificant $(\mathrm{P}>0.05)$, Table 4. 
Table 1: Socio-demographic characteristics of study population by gender

\begin{tabular}{|c|c|c|c|c|}
\hline Variables & Total $(n=2780)$ & Men (n=1447) & Women $(n=1333)$ & P-values \\
\hline $\begin{array}{l}\text { Age } \\
18-24 \\
25-34 \\
35-44 \\
45-54 \\
55-64 \\
\end{array}$ & $\begin{array}{l}329(11.8) \\
368(13.2) \\
734(26.4) \\
767(27.6) \\
582(20.9) \\
\end{array}$ & $\begin{array}{l}188(57.1) \\
170(46.2) \\
279(38.0) \\
432(56.3) \\
378(64.9) \\
\end{array}$ & $\begin{array}{l}141(42.9) \\
198(53.8) \\
455(62.0) \\
335(43.7) \\
204(35.1) \\
\end{array}$ & $<0.001^{* * *}$ \\
\hline $\begin{array}{l}\text { Education } \\
\text { Lower } \\
\text { Higher }\end{array}$ & $\begin{array}{l}1596(57.4) \\
1184(42.6)\end{array}$ & $\begin{array}{l}796(49.9) \\
651(54.0) \\
\end{array}$ & $\begin{array}{l}800(50.1) \\
533(45.0)\end{array}$ & $0.008^{* *}$ \\
\hline $\begin{array}{l}\text { Employment } \\
\text { Employed } \\
\text { Unemployed }\end{array}$ & $\begin{array}{l}1784(64.2) \\
996(35.8)\end{array}$ & $\begin{array}{l}1151(64.5) \\
296(29.7)\end{array}$ & $\begin{array}{l}633(35.5) \\
700(70.3)\end{array}$ & $<0.001^{* * *}$ \\
\hline $\begin{array}{l}\text { Alcohol Intake } \\
\text { Non-user } \\
\text { Regular users }\end{array}$ & $\begin{array}{l}1213(43.6) \\
1567(56.4)\end{array}$ & $\begin{array}{l}579(47.7) \\
868(55.4)\end{array}$ & $\begin{array}{l}634(52.3) \\
699(44.6)\end{array}$ & $<0.001^{* * *}$ \\
\hline $\begin{array}{l}\text { Area of Residence } \\
\text { Rural } \\
\text { Urban }\end{array}$ & $\begin{array}{l}928(33.4) \\
1852(66.6)\end{array}$ & $\begin{array}{l}555(59.8) \\
892(48.2)\end{array}$ & $\begin{array}{l}373(40.2) \\
960(51.8)\end{array}$ & $<0.001^{* * *}$ \\
\hline $\begin{array}{l}\text { Smoking Habits } \\
\text { Non-smokers } \\
\text { Current smokers } \\
\text { Ex-smokers }\end{array}$ & $\begin{array}{l}2512(90.4) \\
79(2.8) \\
189(6.8) \\
\end{array}$ & $\begin{array}{l}1245(49.6) \\
57(72.2) \\
145(76.7) \\
\end{array}$ & $\begin{array}{l}1267(50.4) \\
22(27.8) \\
44(23.3) \\
\end{array}$ & $<0.001^{* * *}$ \\
\hline $\begin{array}{l}\text { Marital Status } \\
\text { Single } \\
\text { Married } \\
\text { Divorced } \\
\end{array}$ & $\begin{array}{l}920(33.1) \\
1785(64.2) \\
75(2.7) \\
\end{array}$ & $\begin{array}{l}627(68.2) \\
798(44.7) \\
22(29.3) \\
\end{array}$ & $\begin{array}{l}293(31.8) \\
987(55.3) \\
53(70.7) \\
\end{array}$ & $<0.001^{* * *}$ \\
\hline $\begin{array}{l}\text { Ethnicity } \\
\text { Ibibio } \\
\text { Annang } \\
\text { Igbo } \\
\text { Yoruba } \\
\text { Hausa }\end{array}$ & $\begin{array}{l}921(33.1) \\
789(28.4) \\
542(19.5) \\
205(7.4) \\
323(11.6) \\
\end{array}$ & $\begin{array}{l}352(38.2) \\
427(54.1) \\
352(64.9) \\
90(43.9) \\
226(70.0) \\
\end{array}$ & $\begin{array}{l}569(61.8) \\
362(45.9) \\
190(35.1) \\
115(56.1) \\
97(30.0) \\
\end{array}$ & $<0.001^{* * *}$ \\
\hline $\begin{array}{l}\frac{\text { Annual Income }}{\text { (Per Annum) }} \\
<120,000 \\
120,000-\$ 250,000 \\
>250,000\end{array}$ & $\begin{array}{l}693(24.9) \\
1668(60.0) \\
419(15.1)\end{array}$ & $\begin{array}{l}291(42.0) \\
883(52.9) \\
273(65.2)\end{array}$ & $\begin{array}{l}402(58.0) \\
78.5(47.1) \\
146(34.8)\end{array}$ & $<0.001^{* * *}$ \\
\hline $\begin{array}{l}\text { Physical Activity } \\
\text { Active } \\
\text { Inactive }\end{array}$ & $\begin{array}{l}953(34.3) \\
1827(65.7)\end{array}$ & $\begin{array}{l}550(57.7) \\
897(49.1)\end{array}$ & $\begin{array}{l}403(42.3) \\
930(50.9)\end{array}$ & $<0.001^{* * *}$ \\
\hline $\begin{array}{l}\text { Family History } \\
\text { Yes } \\
\text { No }\end{array}$ & $\begin{array}{l}956(34.4) \\
1824(65.6)\end{array}$ & $\begin{array}{l}438(45.8) \\
1009(55.3)\end{array}$ & $\begin{array}{l}518(54.2) \\
815(44.7)\end{array}$ & $<0.001^{* * *}$ \\
\hline
\end{tabular}

Values in the parenthesis are percentages, ${ }^{* *} \mathrm{P}<0.01$ significant at $1 \%,{ }^{* * *} \mathrm{P}<0.001$ significant at $0.1 \%$. 
Table 2: Prevalence of non-communicable diseases in relation to risk factor by gender

\begin{tabular}{|c|c|c|c|c|c|c|c|}
\hline \multirow[b]{2}{*}{ Risk Factors } & \multicolumn{2}{|c|}{ Males (n=1447) } & \multicolumn{2}{|c|}{ Females $(n=1333)$} & \multirow[b]{2}{*}{$\begin{array}{l}\text { All With } \\
\text { NCDs (n } \\
=912)\end{array}$} & \multirow[b]{2}{*}{$\begin{array}{lr}\text { All Without } & \text { W } \\
\text { NCDs } \quad(n= \\
1868)\end{array}$} & \multirow[b]{2}{*}{ P-values } \\
\hline & $\begin{array}{l}\text { With NCDs } \\
(n=434)\end{array}$ & $\begin{array}{l}\text { Without } \\
\text { NCDs } \\
(n=1013)\end{array}$ & $\begin{array}{l}\text { With } \\
\text { NCDs } \\
(n=478)\end{array}$ & $\begin{array}{l}\text { Without } \\
\text { NCDs } \\
\text { (n= 855) }\end{array}$ & & & \\
\hline $\begin{array}{l}\text { Age } \\
18-24 \\
25-34 \\
35-44 \\
45-54 \\
55-64\end{array}$ & $\begin{array}{l}9(4.8) \\
17(10.0) \\
50(17.9) \\
201(46.5) \\
157(41.5)\end{array}$ & $\begin{array}{l}179(95.2) \\
153(90.0) \\
229(82.1) \\
231(53.5) \\
221(58.5)\end{array}$ & $\begin{array}{l}7(5.0) \\
24(12.1) \\
86(18.9) \\
228(68.1) \\
133(65.2)\end{array}$ & $\begin{array}{l}134(95.0) \\
174(87.9) \\
369(81.1) \\
107(31.9) \\
71(34.8)\end{array}$ & $\begin{array}{l}16(1.8) \\
41(4.5) \\
136(14.9) \\
429(47.0) \\
290(31.8)\end{array}$ & $\begin{array}{l}313(16.8) \\
327(17.5) \\
598(32.0) \\
338(18.1) \\
292(15.6)\end{array}$ & $<0.001^{* * *}$ \\
\hline $\begin{array}{l}\text { Dietary Habit } \\
\text { Good } \\
\text { Poor }\end{array}$ & $\begin{array}{l}196(28.2) \\
238(31.6) \\
\end{array}$ & $\begin{array}{l}499(71.8) \\
514(68.4) \\
\end{array}$ & $\begin{array}{l}238(32.4) \\
240(40.1) \\
\end{array}$ & $\begin{array}{l}496(67.6) \\
359(59.9) \\
\end{array}$ & $\begin{array}{l}434(47.6) \\
478(25.4) \\
\end{array}$ & $\begin{array}{l}995(53.3) \\
873(46.7) \\
\end{array}$ & 0.006 \\
\hline $\begin{array}{ll}\text { Area } & \text { of } \\
\text { Residence } & \\
\text { Rural } \\
\text { Urban }\end{array}$ & $\begin{array}{l}156(28.1) \\
278(31.2) \\
\end{array}$ & $\begin{array}{l}399(71.9) \\
614(68.8) \\
\end{array}$ & $\begin{array}{l}102(27.3) \\
376(39.2) \\
\end{array}$ & $\begin{array}{l}271(72.7) \\
584(60.8) \\
\end{array}$ & $\begin{array}{l}258(28.3) \\
654(71.7) \\
\end{array}$ & $\begin{array}{l}670(35.9) \\
1198(64.1) \\
\end{array}$ & $<0.001^{* * *}$ \\
\hline $\begin{array}{l}\text { Physical } \\
\text { Activity } \\
\text { Active } \\
\text { Inactive }\end{array}$ & $\begin{array}{l}134(24.4) \\
300(50.3)\end{array}$ & $\begin{array}{l}416(75.6) \\
297(49.7)\end{array}$ & $\begin{array}{l}102(25.3) \\
376(40.4)\end{array}$ & $\begin{array}{l}301(74.7) \\
554(59.6)\end{array}$ & $\begin{array}{l}236(25.9) \\
676(74.1)\end{array}$ & $\begin{array}{l}717(38.4) \\
1151(61.6)\end{array}$ & $<0.001^{* * *}$ \\
\hline $\begin{array}{l}\text { Education } \\
\text { Lower } \\
\text { Higher }\end{array}$ & $\begin{array}{l}117(14.7) \\
317(48.7) \\
\end{array}$ & $\begin{array}{l}679(85.3) \\
334(51.3) \\
\end{array}$ & $\begin{array}{l}125(15.6) \\
353(66.2) \\
\end{array}$ & $\begin{array}{l}675(84.4) \\
180(33.8) \\
\end{array}$ & $\begin{array}{l}242(26.5) \\
670(73.5) \\
\end{array}$ & $\begin{array}{l}1354(72.5) \\
514(27.5)\end{array}$ & $<0.001^{* * *}$ \\
\hline $\begin{array}{l}\frac{\text { Alcohol }}{\text { Consumption }} \\
\text { Non-users } \\
\text { Users }\end{array}$ & $\begin{array}{l}226(39.0) \\
208(31.5)\end{array}$ & $\begin{array}{l}353(61.0) \\
452(68.5)\end{array}$ & $\begin{array}{l}236(37.2) \\
242(34.6)\end{array}$ & $\begin{array}{l}398(62.8) \\
457(65.4)\end{array}$ & $\begin{array}{l}462(50.7) \\
450(49.3)\end{array}$ & $\begin{array}{l}905(48.4) \\
963(51.5)\end{array}$ & $0.010^{*}$ \\
\hline $\begin{array}{l}\text { Family History } \\
\text { Yes } \\
\text { No }\end{array}$ & $\begin{array}{l}223(50.9) \\
208(31.5) \\
\end{array}$ & $\begin{array}{l}215(49.1) \\
798(79.1)\end{array}$ & $\begin{array}{l}246(47.5) \\
232(28.5) \\
\end{array}$ & $\begin{array}{l}272(52.5) \\
583(71.5) \\
\end{array}$ & $\begin{array}{l}469(51.4) \\
443(48.6)\end{array}$ & $\begin{array}{l}487(26.1) \\
1381(73.9)\end{array}$ & $<0.001^{* * *}$ \\
\hline $\begin{array}{l}\text { BMI } \\
\text { Obese } \\
\text { Not Obese }\end{array}$ & $\begin{array}{l}229(76.6) \\
205(17.9) \\
\end{array}$ & $\begin{array}{l}70(23.4) \\
943(82.1) \\
\end{array}$ & $\begin{array}{l}305(77.0) \\
173(18.5) \\
\end{array}$ & $\begin{array}{l}91(23.0) \\
764(81.5) \\
\end{array}$ & $\begin{array}{l}534(58.6) \\
378(41.4) \\
\end{array}$ & $\begin{array}{l}161(8.6) \\
1707(91.4)\end{array}$ & $<0.001^{* * *}$ \\
\hline $\begin{array}{l}\text { WC } \\
\text { Normal } \\
\text { Abnormal }\end{array}$ & $\begin{array}{l}209(21.2) \\
225(48.6) \\
\end{array}$ & $\begin{array}{l}775(78.8) \\
238(51.4) \\
\end{array}$ & $\begin{array}{l}221(30.1) \\
257(42.9) \\
\end{array}$ & $\begin{array}{l}513(69.9) \\
342(57.1) \\
\end{array}$ & $\begin{array}{l}430(47.1) \\
482(52.9) \\
\end{array}$ & $\begin{array}{l}1288(68.9) \\
580(31.0) \\
\end{array}$ & $<0.001^{* * *}$ \\
\hline $\begin{array}{l}\text { Smoking Habit } \\
\text { Non-smokers } \\
\text { Current } \\
\text { smokers } \\
\text { Ex-smokers }\end{array}$ & $\begin{array}{l}401(32.2) \\
15(26.3) \\
18(12.4)\end{array}$ & $\begin{array}{l}844(67.8) \\
42(73.7) \\
127(87.6)\end{array}$ & $\begin{array}{l}465(36.7) \\
4(18.2) \\
9(20.5)\end{array}$ & $\begin{array}{l}802(63.3) \\
18(81.8) \\
35(79.5)\end{array}$ & $\begin{array}{l}866(95.0) \\
19(2.1) \\
27(2.9)\end{array}$ & $\begin{array}{l}1646(88.1) \\
60(3.2) \\
162(85.7)\end{array}$ & $<0.001^{* * *}$ \\
\hline $\begin{array}{l}\text { Work Stress } \\
\text { High } \\
\text { Low }\end{array}$ & $\begin{array}{l}295(33.4) \\
139(24.6) \\
\end{array}$ & $\begin{array}{l}588(66.6) \\
425(75.4) \\
\end{array}$ & $\begin{array}{l}347(41.1) \\
131(26.8) \\
\end{array}$ & $\begin{array}{l}498(58.9) \\
357(73.2) \\
\end{array}$ & $\begin{array}{l}642(70.4) \\
270(29.6) \\
\end{array}$ & $\begin{array}{l}1086(58.1) \\
782(41.9)\end{array}$ & $<0.001^{* * *}$ \\
\hline $\begin{array}{l}\text { Dyslipidemia } \\
\text { High TC } \\
\text { High TG } \\
\text { High LDL } \\
\text { Low HDL }\end{array}$ & $\begin{array}{l}145(84.3) \\
182(83.9) \\
207(79.9) \\
301(91.2)\end{array}$ & $\begin{array}{l}27(15.7) \\
35(16.1) \\
52(20.1) \\
29(8.8)\end{array}$ & $\begin{array}{l}185(92.5) \\
198(94.7) \\
201(93.9) \\
297(95.5)\end{array}$ & $\begin{array}{l}15(7.5) \\
11(5.3) \\
13(6.1) \\
4(4.5)\end{array}$ & $\begin{array}{l}330(36.2) \\
380(41.7) \\
408(44.7) \\
598(65.6)\end{array}$ & $\begin{array}{l}42(2.2) \\
46(2.5) \\
65(3.5) \\
33(1.8)\end{array}$ & $<0.001^{* * *}$ \\
\hline
\end{tabular}

Values in the parenthesis are percentages,

${ }^{*} \mathrm{P}<0.05$ significant $5 \%,{ }^{* *} \mathrm{P}<0.01$ significant at $1 \%,{ }^{* * *} \mathrm{P}<0.001$ significant at $0.1 \%$. 
Table 3: Age and Sex Specific Prevalence of Non-communicable Diseases

\begin{tabular}{|c|c|c|c|c|c|c|c|c|c|c|c|c|c|}
\hline \multirow[b]{2}{*}{ NCDs } & \multicolumn{5}{|c|}{ Men in Different Age Groups } & \multicolumn{5}{|c|}{ Women in Different Age Groups } & \multicolumn{3}{|c|}{ Overall Total Prevalence } \\
\hline & $18-24$ & $25-34$ & $35-44$ & $45-54$ & $55-64$ & $18-24$ & $25-34$ & $35-44$ & $45-54$ & $55-64$ & $\begin{array}{l}\text { Men } \\
(n=1447)\end{array}$ & $\begin{array}{l}\text { Women } \\
(\mathrm{N}=1337)\end{array}$ & P-values \\
\hline$\frac{\text { Obesity }}{(n=695)} 25 \%$ & $\begin{array}{l}36 \\
(12.0)\end{array}$ & $\begin{array}{l}49 \\
(16.4)\end{array}$ & $\begin{array}{l}55 \\
(18.4)\end{array}$ & $\begin{array}{l}70 \\
(23.4)\end{array}$ & $\begin{array}{l}89 \\
(29.8)\end{array}$ & $\begin{array}{l}53 \\
(13.4)\end{array}$ & $\begin{array}{l}69 \\
(17.4)\end{array}$ & $\begin{array}{l}81 \\
(20.5)\end{array}$ & $\begin{array}{l}101 \\
(25.5)\end{array}$ & $\begin{array}{l}92 \\
(23.2)\end{array}$ & $\begin{array}{l}299 \\
(20.7)\end{array}$ & $\begin{array}{l}396 \\
(29.7)\end{array}$ & $<0.001^{* * *}$ \\
\hline $\begin{array}{l}\text { Hypertension } \\
(\mathrm{n}=401) \\
14.4 \% \\
\end{array}$ & $\begin{array}{l}5 \\
(2.7)\end{array}$ & $\begin{array}{l}13 \\
(7.1)\end{array}$ & $\begin{array}{l}35 \\
(19.1)\end{array}$ & $\begin{array}{l}59 \\
(32.2)\end{array}$ & $\begin{array}{l}71 \\
(38.8)\end{array}$ & $\begin{array}{l}9 \\
(5.6)\end{array}$ & $\begin{array}{l}18 \\
(11.1)\end{array}$ & $\begin{array}{l}32 \\
(19.8)\end{array}$ & $\begin{array}{l}45 \\
(27.8)\end{array}$ & $\begin{array}{l}58 \\
(35.8)\end{array}$ & $\begin{array}{l}183 \\
(12.6)\end{array}$ & $\begin{array}{l}162 \\
(12.2)\end{array}$ & $<0.001^{* * *}$ \\
\hline $\begin{array}{l}\frac{\text { Diabetes }}{\text { Mellitus }} \\
\frac{(\mathrm{n}=354)}{12.7 \%}\end{array}$ & $\begin{array}{l}6 \\
(4.3)\end{array}$ & $\begin{array}{l}8 \\
(5.7)\end{array}$ & $\begin{array}{l}25 \\
(17.7)\end{array}$ & $\begin{array}{l}48 \\
(34.0)\end{array}$ & $\begin{array}{l}54 \\
(38.3)\end{array}$ & $\begin{array}{l}10 \\
(4.7)\end{array}$ & $\begin{array}{l}14 \\
(6.6)\end{array}$ & $\begin{array}{l}51 \\
(23.9)\end{array}$ & $\begin{array}{l}67 \\
(31.5)\end{array}$ & $\begin{array}{l}71 \\
(33.3)\end{array}$ & $\begin{array}{l}141 \\
(9.7)\end{array}$ & $\begin{array}{l}213 \\
(16.0)\end{array}$ & $<0.001^{* * *}$ \\
\hline $\begin{array}{l}\text { M.S. } \\
\frac{\text { Disorder }}{=556) 20 \%}\end{array}$ & $\begin{array}{l}21 \\
(10.4)\end{array}$ & $\begin{array}{l}31 \\
(15.3)\end{array}$ & $\begin{array}{l}47 \\
(23.3)\end{array}$ & $\begin{array}{l}54 \\
(26.7)\end{array}$ & $\begin{array}{l}49 \\
(24.3)\end{array}$ & $\begin{array}{l}35 \\
(9.9)\end{array}$ & $\begin{array}{l}51 \\
(14.4)\end{array}$ & $\begin{array}{l}84 \\
(23.7)\end{array}$ & $\begin{array}{l}90 \\
(25.4)\end{array}$ & $\begin{array}{l}94 \\
(26.6)\end{array}$ & $\begin{array}{l}202 \\
(14.0)\end{array}$ & $\begin{array}{l}354 \\
(26.7)\end{array}$ & $<0.001^{* * *}$ \\
\hline $\begin{array}{l}\frac{\text { Resp. }}{\text { Disorders }} \\
(n=281) \\
10.1 \%\end{array}$ & $\begin{array}{l}4 \\
(3.2)\end{array}$ & $\mid \begin{array}{l}12 \\
(9.7)\end{array}$ & $\begin{array}{l}29 \\
(23.4)\end{array}$ & $\begin{array}{l}43 \\
(34.7)\end{array}$ & $\begin{array}{l}36 \\
(29.0)\end{array}$ & $\begin{array}{l}7 \\
(6.9)\end{array}$ & $\begin{array}{l}13 \\
(12.9)\end{array}$ & $\begin{array}{l}38 \\
(20.8)\end{array}$ & $\begin{array}{l}27 \\
(26.7)\end{array}$ & $\begin{array}{l}32 \\
(32.7)\end{array}$ & $\begin{array}{l}124 \\
(8.6)\end{array}$ & $\begin{array}{l}101 \\
(7.6)\end{array}$ & $<0.001^{* * *}$ \\
\hline Total & & & & & & & & & & & & & \\
\hline
\end{tabular}

Values in the parenthesis are percentages, ${ }^{* * *} \mathrm{P}<0.001$ significant at 0.001 alpha level.

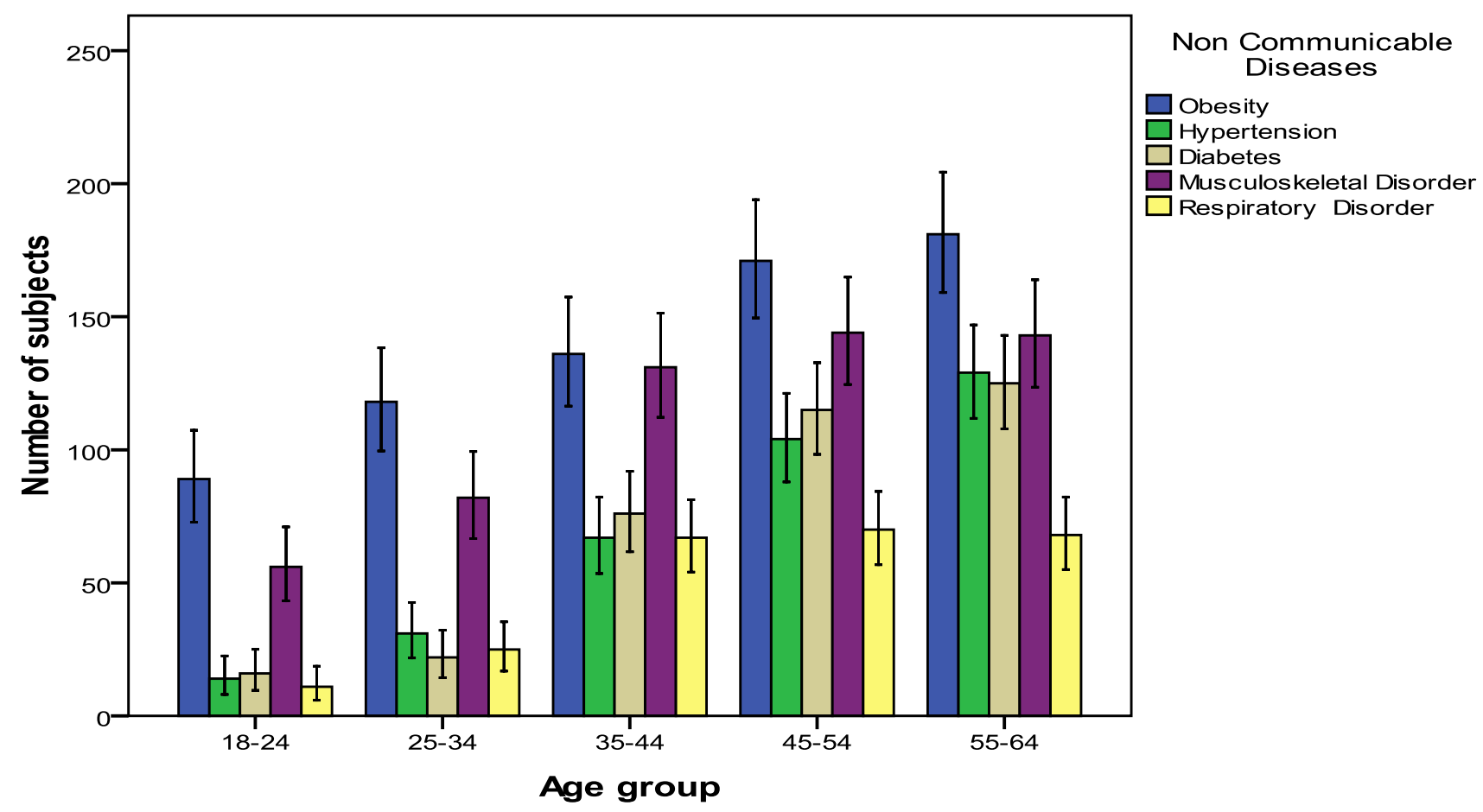

Figure 1: The distribution of NCDs in relation to age 
Table 4: Multiple logistic regression showing association between NCDs and its risk factors (adjusted odd ratios and $\mathbf{9 5 \%}$ confidence interval)

\begin{tabular}{|c|c|c|c|c|c|c|}
\hline \multirow[b]{2}{*}{ Risk Factors } & \multicolumn{3}{|l|}{ Male } & \multicolumn{3}{|l|}{ Female } \\
\hline & $\begin{array}{l}\text { Adjusted } \\
\text { Odd ratios }\end{array}$ & 95\% C.I & P-values & $\begin{array}{l}\text { Adjusted } \\
\text { Odd ratios }\end{array}$ & 95\% C.I & P-values \\
\hline $\begin{array}{l}\text { Age } \\
\text { Young Adults }(\leq 44) \\
\text { Old Adults }(\geq 45)\end{array}$ & $\begin{array}{l}1.0 \quad \text { (reference) } \\
4.78\end{array}$ & $3.33-10.60$ & $<0.001^{* * *}$ & 5.02 & $4.83-12.74$ & $<0.001^{* * *}$ \\
\hline $\begin{array}{l}\text { Dietary Habits } \\
\text { Good } \\
\text { Poor }\end{array}$ & $\begin{array}{l}1.00 \text { (reference) } \\
4.23\end{array}$ & $1.641-10.906$ & $0.003^{* *}$ & $\begin{array}{l}1.00 \\
\text { (reference) } \\
4.96\end{array}$ & $1.893-12.988$ & $0.001^{* *}$ \\
\hline $\begin{array}{l}\text { Smoking Habits } \\
\text { Non-smokers } \\
\text { Current smokers } \\
\text { Ex-smokers }\end{array}$ & $\begin{array}{ll}1.0 & \text { (reference) } \\
0.970 & \\
0.82 & \end{array}$ & $\begin{array}{l}0.767-1.227 \\
0.627-1.058\end{array}$ & $\begin{array}{l}0.800^{\mathrm{NS}} \\
0.125^{\mathrm{NS}}\end{array}$ & $\begin{array}{l}1.00 \\
\text { (reference) } \\
1.02 \\
1.06 \\
\end{array}$ & $\begin{array}{l}0.824-1.346 \\
0.926-1.528\end{array}$ & $\begin{array}{l}0.650^{\mathrm{NS}} \\
0.823^{\mathrm{NS}}\end{array}$ \\
\hline $\begin{array}{l}\text { Physical Activity } \\
\text { Active } \\
\text { Inactive }\end{array}$ & $\begin{array}{ll}1.0 & \text { (reference) } \\
2.08 & \end{array}$ & $1.595-2.722$ & $<0.001^{* * *}$ & $\begin{array}{l}1.00 \\
\text { (reference) } \\
3.04\end{array}$ & $2.271-4.062$ & $<0.001^{* * *}$ \\
\hline $\begin{array}{l}\text { Alcohol } \\
\text { Consumption } \\
\text { Non-users } \\
\text { Regular users } \\
\end{array}$ & $\begin{array}{ll}1.0 & \text { (reference) } \\
0.913 & \end{array}$ & $\begin{array}{l}1.0 \quad \text { (reference) } \\
0.626-1.331\end{array}$ & $0.634^{\mathrm{NS}}$ & $\begin{array}{l}1.00 \\
\text { (reference) } \\
1.26 \\
\end{array}$ & $0.895-1.766$ & $0.188^{\mathrm{NS}}$ \\
\hline 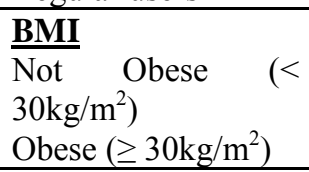 & $\begin{array}{ll}1.0 & \text { (reference) } \\
2.76 & \end{array}$ & $1.856-4.094$ & $<0.001^{* * *}$ & $\begin{array}{l}1.00 \\
\text { (reference) } \\
3.72 \\
\end{array}$ & $2.525-5.470$ & $<0.001^{* * *}$ \\
\hline $\begin{array}{l}\frac{\text { WC }}{\text { Normal }} \\
\text { Abnormal }\end{array}$ & $\begin{array}{ll}1.0 & \text { (reference) } \\
0.913 & \\
\end{array}$ & $0.626-1.331$ & $0.634^{\mathrm{NS}}$ & 1.65 & $1.182-2.625$ & $<0.003^{* *}$ \\
\hline $\begin{array}{l}\text { Area of Residence } \\
\text { Rural } \\
\text { Urban }\end{array}$ & $\begin{array}{ll}1.0 & \text { (reference) } \\
1.53 & \\
\end{array}$ & $1.162-2.021$ & $0.002^{* *}$ & 1.85 & $1.285-3.084$ & $0.028^{*}$ \\
\hline $\begin{array}{l}\text { Work Stress } \\
\text { High } \\
\text { Low }\end{array}$ & $\begin{array}{ll}1.0 & \text { (reference) } \\
2.81 & \end{array}$ & $1.899-4.163$ & $<0.001^{* * *}$ & $\begin{array}{l}1.00 \\
\text { (reference) } \\
5.23\end{array}$ & $3.332-8.214$ & $<0.001^{* * *}$ \\
\hline $\begin{array}{l}\text { Dyslipidemia } \\
\text { TC } \\
\text { (Abnormal/Normal) } \\
\text { TG } \\
\text { (Abnormal/Normal) } \\
\text { LDL } \\
\text { (Abnormal/Normal) } \\
\text { HDL } \\
\text { (Abnormal/Normal) }\end{array}$ & $\begin{array}{l}1.51 \\
1.46 \\
1.48 \\
1.04\end{array}$ & $\begin{array}{l}1.198-1.899 \\
1.096-1.956 \\
0.975-2.260 \\
0.693-1.548\end{array}$ & $\begin{array}{l}<0.001^{* * *} \\
0.001^{* *} \\
0.066^{\mathrm{NS}} \\
0.864^{\mathrm{NS}}\end{array}$ & $\begin{array}{l}2.17 \\
1.38 \\
0.953 \\
0.953\end{array}$ & $\begin{array}{l}1.475-3.184 \\
1.015-1.875 \\
0.408-2.226 \\
0.408-2.226\end{array}$ & $\begin{array}{l}<0.001^{* * *} \\
0.04^{*} \\
0.911^{\mathrm{NS}} \\
0.911^{\mathrm{NS}}\end{array}$ \\
\hline $\begin{array}{l}\text { Education } \\
\text { Lower } \\
\text { Higher }\end{array}$ & $\begin{array}{ll}1.0 & \text { (reference) } \\
1.75 & \end{array}$ & $1.391-2.207$ & $<0.001^{* * *}$ & $\begin{array}{l}1.00 \\
\text { (reference) } \\
1.29\end{array}$ & $1.002-1.650$ & $0.049^{* * *}$ \\
\hline $\begin{array}{l}\text { Family History } \\
\text { No } \\
\text { Yes } \\
\end{array}$ & $\begin{array}{ll}1.0 & \text { (reference) } \\
4.18 & \\
\end{array}$ & $3.51-4.970$ & $<0.001^{* * *}$ & 4.16 & $3.455-5.019$ & $<0.001^{* * *}$ \\
\hline
\end{tabular}


Adjusted for marital status, ethnicity, employment status and annual income.

NS:P $>0.05$ not significant at $1 \%, ;{ }^{* * *} \mathrm{P}<0.001$ significant at $0.1 \%$.

\section{Discussion}

This study was conducted to evaluate the age and sex specific burden and risk factors of non-communicable diseases in a mixed population of urban and rural dwellers in Southern part of Nigeria.

The main findings were that; the overall prevalence of non-communicable diseases (NCDs) was 32.8\%. Prevalence of obesity, musculoskeletal disorders (MSDs), hypertension, diabetes mellitus and chronic respiratory disorders were as follows: $25 \%, 20.1 \%, 14.4 \%, 12.7 \%$ and $10 \%$ respectively. Variation in prevalence was recorded in different age groups and gender with preponderance of cases in females and older participants.

Also, result of univariate analysis showed that, prevalence of NCDs was significantly associated with four particular behaviors, physical inactivity, unhealthy diet, smoking habits and excessive alcohol intake. Age, positive family history, educational status, area of residence and generalized obesity were also significantly associated with increase prevalence of NCDs.

The high prevalence of NCDs as recorded in this survey is indicative of the increasing trend of the disease burden in Nigeria, a sub-Saharan African country, which together with already overwhelmed burden of infectious diseases and malnutrition is posing a double burden to the health and economy of this region (Lopez et al. 2006). Also, the prevalence of 32.8\% as recorded in this survey lend support to the projection from the Global Burden of Diseases study which suggested that by the year 2020 the proportion of the overall burden in subSaharan Africa due to NCDs will increase to somewhere between $26 \%$ and 34\%, and among adults aged 15-59 years to between 37\% and 42\% (Murray et al 1996). It has heralded the higher probability of death from NCDs in sub-Saharan Africa than the Established Market Economies (Unwin et al. 2001), thus underscoring the need for immediate effort to halt the trend.

The prevalence of NCDs as recorded here is comparable to values obtained in some developed countries of the world (Anderson et al. 2009). The implication of this is that, the trend in Nigeria and other developing countries of sub-Saharan Africa is growing rapidly to catch up with the developed nations of the world regardless of the marked socio-economic disparities between the two regions. Such high prevalence of NCDs reflects the cumulative effect of unhealthy life style and risk factors of people's life span, life style habits such as; poor dietary habits, physical inactivity, smoking habits, and alcohol abuse are strongly 
implicated. These are known features of urbanization and economic transition now found in most countries of sub-Saharan Africa including Nigeria (Cooper et al. 1997; Kruger et al. 2002).

They are associated with increase prevalence of overweight and obesity known to act as pacesetters to other NCDs (Ziraba et al. 2009). A well established body of literature documents a strong linkage between obesity and other NCDs such as; type 2 diabetes mellitus (James et al. 2003; Meisinger et al. 2006), hypertension (WHO, 2000; Stamler, 2010; Nwachukwu, 2010), dyslipidemia (WHO, 2000), chronic respiratory disorders and cancers. This effect is mediated partly by causing insulin resistance which occupies the central position in the pathogenesis of NCDs and also is highly atherogenic (WHO, 2000; Fontains et al. 2003; Peters et al. 2003).

In our study participants, the effects of these life style characteristics on prevalent NCDs were very obvious. Physical inactivity, unhealthy diet, smoking habits and alcohol abuse were associated with increase odds for prevalent NCDs.

This also explains the high prevalence of obesity recorded in this survey which has reinforced the observation that obesity is on increase in urban and rural areas of sub-Saharan Africa (Ziraba et al. 2009) and signal the impending epidemic of communicable and non-communicable diseases in this region in the near future (WHO, 2005). Regrettably, the prevalence of NCDs in this survey could not be compared with values from most countries of sub-Saharan African regions because most African countries have not conducted their risk factors survey to establish the national base line prevalence rate and accurately quantify the magnitude of the problem. Some countries in this region make use of data from health management information system to estimate their national prevalence status (Mufunda et al. 2006).

Also some studies carried out in this region are characterized by: variations in case definition, data collection and diagnosing protocols. For example, variations in cut off limits for diagnosing hypertension, diabetes mellitus and obesity have been observed in some studies making such results un-comparable between studies. However, the mostly used method for quantifying the burden of NCDs worldwide is the approach that measures the country regional and global NCDs in terms of Disability Adjusted Life Years (DALYs) (Boutayed et al. 2005). Data from such computation is either scanty or unavailable in countries of sub-Saharan Africa because of the lack of reliable mortality and morbidity data (Murray et al. 1997). This is evidenced by Country estimates of NCDs mortality and selected risk factors 2008, documented in the 2010 Global status report on NCDs by WHO which was characterized by a high degree of uncertainty for some countries of sub-Saharan Africa including Nigeria because they were not based on any national NCD mortality data, rather the estimates for these countries were based 
on a combination of country life tables, cause of death models, regional causes of death patterns and WHO and UNAIDS programme estimates for some major causes of death (not including NCDs) (WHO, 2010).

Like previous studies (MOHSS, 1997; Amoah et al. 2002; Thankappan et al. 2010), a significant association between age and prevalence of NCDs was observed. Older age was associated with 4.78 odds for prevalent NCDs in males and 5.0 odds in females. They opined that, most chronic diseases are present at this period of life because of the interactions between multiple disease processes as well as more general losses in physiological function (Darton-Hill et al. 2001). Age is associated with decline in glucose tolerance, insulin sensitivity and insulin secretion (Chang et al. 2003). Insulin resistance at old age has been strongly implicated and extensively evaluated (Facchini et al. 2001). A study to evaluate the ability of age related diseases, demonstrated that age related insulin resistance was an independent predictor of all clinical events.

However, age per se is not a constant finding and thus insulin resistance among older individuals could not be entirely attributed to old age. Miszkurka et al, (2012) observed a markedly high prevalence of NCDs in young adults of Burkina Faso. Similar findings have been reported in Tanzania and South Asia (Unwin et al. 2001; Sharma et al. 2005). Boden et al, (1993) demonstrated in their study that whole body sensitivity to insulin was not significantly different in elderly compared with young men, although elderly men tended to have rates of glucose uptake, oxidation and storage that were slightly lower than that of the younger. Of particular interest was the observation that all parameters of glucose metabolism (glucose uptake, oxidation and storage) correlated well with body fat composition and distribution, but not so to differences in age. Fink et al (1983) demonstrated that decline in peripheral glucose uptake among a group of elderly men and women correlated more with increase total body fat/mass. Rowe et al (1983), in their study found that a lower glucose infusion rate was needed to maintain the euglycemic state in the elderly at equal insulin concentration with the younger individuals.

Other studies have offered explanation to this disparity. In one study, it was opined that, obesity and physical inactivity could account for the differences in glucose tolerance observed in older people. Also, effect of age was still obvious at older age of 60-92 years. These observations corroborate with findings of this study. Non-communicable diseases were more common among the older than young participants ( $\mathrm{OR}=4.78$ and 5.02 for male and females respectively). General obesity (as depicted by $\mathrm{BMI}$ ) was significantly associated $(\mathrm{OR}=2.72$ ) with prevalence of NCDs in the elderly than the young participants.

The foregoing research findings have underscored the significant position occupied by the entire body fat composition to prevalence of NCDs. As 
previously documented, obesity causes insulin resistance which in turns trigger on the development of NCDs such as: diabetes mellitus, hypertension (Gryglewska et al. 1995), cardiovascular diseases (Bergstrom et al. 2001), dyslipidemia (Folsom et al. 2000), cancers (Bergstrom et al. 2001), chronic respiratory disorders and musculoskeletal disorders. Diabetes mellitus is 3-7 times more prevalent in the obese than normal weight adult and those with BMI $\geq 35$ are 20 times more likely to develop NCDs than those with BMI between 18.5 and 24.9 (Mokdad et al. 2001).

An increasing body of evidence shows that mortality from obesity associated health problems increases exponentially with body weight, for example, cardiovascular disease risk is double if BMI is $>25$ and nearly quadrupled if the $\mathrm{BMI}$ is $>29$, the risk of developing diabetes was 40 times with BMI $>35$ (Mohammed, 2003).

Findings of this survey therefore provide a fresh empiric support for a long held concept that if obesity risk factors such as poor dietary habits, physical inactivity, smoking habits and alcohol abuse are under strict control, a concomitant remarkable reduction in prevalence of NCDs will ensue even in a genetically predisposed individual (Tehard et al. 2006).

Poor dietary habit was associated with 4.23 and 4.96 odds for prevalent NCDs in males and females respectively. Similarly, physical inactivity, current smoking habits (female), excessive alcohol consumption (female) and work stress were all associated with higher odds for prevalent NCDs. The plausibility of this research findings have been supported by previous documentations (Khatib, 2004).

Consistent with previous studies (Misra et al. 2006; Msyamboza et al. 2011), gender disparity was obvious in this study. More women than men were exposed to several risk factors of NCDs and suffered NCDs than men. They were found in the lower socioeconomic class as depicted by their lower educational status, higher rate of unemployment and low annual income. A well established body of literature, documents a strong linkage between factors that depict the socioeconomic status of individuals as mentioned above and their health awareness and practices. Socioeconomically disadvantaged populations are more likely to be unaware and strongly affected by health problems such as diabetes mellitus, cancers, hypertension and respiratory disorders (Cesaroni et al. 2003; Dalstra et al. 2005; Ellison-Loschmann et al. 2007).

Also, obesity was more pronounced in women than men, probably because they were more physically inactive and physiologically predisposed. (Jovanavic et al. 1996; Prasonna, 1996; Grant et al. 2009).

Furthermore, several previous studies have reported an association between NCDs risk factors and urban/rural area of residence, with overweight/ obesity and physical inactivity being more frequent in urban than rural areas of sub- 
Saharan Africa (Belue et al. 2009; Msyamboza et al. 2011). Such findings are congruent with the results of this survey. More women resided in urban areas with associated high level of physical inactivity and overweight/obesity and therefore higher prevalence of NCDs.

The higher frequency of the risk factors in women than men attenuates the insulin sensitivity advantage of women over men and therefore predisposes them to higher odds for prevalent NCDs as observed in this study. However this is not a universal finding, contrasting results have been recorded in some other studies Msyamboza et al, (2011), in their study in Malawi observed that tobacco smoking, alcohol consumption and raised blood pressure were more frequent in men than women whereas overweight, obesity and raised cholesterol were more frequent in females than males.

The research findings also highlighted a significant association between positive family history and prevalence of NCDs. Participants with positive family history of NCDs had 4.18 and 4.16 odds for prevalent NCDs in males and females respectively. This observation lend support to the observation that offspring born to mothers with diabetes mellitus have three times higher risk of developing type 2 diabetes in later life (Thiam et al. 2006). A higher risk is observed if both parents are diabetics. The relationship between T2DM in family members and eventual onset in children and later life is so close that some researchers are calling for children living in areas experiencing epidemic of diabetes to be considered at risk (Pinhas et al. 2005). Two factors have been reported to affect this relationship. These include: the age when first diagnosed and the type of diabetes (Vander Sande et al. 2001). Similarly, positive family history of hypertension increases the odds to 4 times (Corvol et al. 1992), while for obesity the likelihood is about 3.1 times (Mo-suwan et al. 1996).

The strength and precisions of this study were obtained from the large sample size which gave a fair representation of the studied area. Also variables under study (anthropometric and non anthropometric) were actually measured. This eliminated errors and inaccuracies due to self reported variables. Limitations encountered in the study were: inability to assess in order to determine the prevalence of cancers in the study, which could have affected the overall prevalence rate of NCDs in this survey.

Conclusion: The prevalence of NCDs was high and was influenced by several modifiable and un-modifiable risk factors. Intervention programmes should focus on these factors to put a halt and reverse the trend.

Acknowledgement: We have acknowledged the priceless effort put in by other members of the study team: the paramedical staff, the technical crew, the statisticians and Joshua the chief computer operator for their unrelenting effort. 
Funding: This research received no specific grant from any funding agency Competing interest: None.

\section{REFERENCES}

1. Amoah AG, Owusu SK, Adjei S (2002). Diabetes in Ghana: a community based prevalence study in Greater Accra. Diabetes Res Clin Pract, 56 (3): 197-205.

2. Anderson GF, PiHman P, Herbert R, Das K (2009). Non-communicable Chronic Diseases in Latin America and Caribbean. U.S Agency for Internal Development (USAID), 1-55.

3. Aspray TJ, Mugusi F, Rashid S, Whiting D, Edwards R, Alberti K and Unwin N (2000). Rural and urban differences in diabetes prevalence in Tanzania: the role of obesity, physical inactivity and urban living. Transactions of the Royal Society of Tropical Medicine and Hygiene, 94 (6): 637-644.

4. Belue R, Okoror TA, Iwelunmor J, Taylor KD, Degboe AN (2009). An overview of cardiovascular risk factor burden in sub-Saharan Africa countries: a sociocultural perspective. Global Health, 5:10.

5. Berger GM and Mardis AD (2002). Diagnosis, management and prevention of the common dyslipemia in South Africa clinical guidelines. South African Medical Journal, 90:164-178.

6. Bergstrom A, Pisani P, Tenet V, et al (2001). Overweight as an avoidable cause of cancer in Europe. Int J Cancer, 91: 421-430.

7. Boden G, Chen X, Desantis RA, Kendrick Z (1993). Effects of age and body fat on insulin resistance in healthy men. Diabetes Care, 16(5):728-733.

8. Bourne LT, Lambert EV, and Steyn K (2002). Where does the black population of South Africa stand on the nutrition transition? Public Health and Nutrition, 5(1A):157-162.

9. Boutayeb A and Boutayeb S (2005). The burden of non-communicable diseases in developing countries. International Journal of Equity and Health, 5:2.doi: 10.1186/1475-9276-4-4. Available

from http://www.equityhealthj.com/content/4/1/2.

10. Cesaroni $\mathrm{G}$, et al (2003). Individual and area-based indicators of socio-economic status and childhood asthma.TheEuropean Respiratory Journal, 22:619-624.

11. Chang AM, Halter JB (2003). Aging and insulin secretion. Am J Physiol Endocrinol Meta, 284:E7-E12

12. Chobanian AV, Bakris GL, Black HR, Cushman WC, Green LA, Izzo JL Jr, (2003). The seventh report of the Joint National Committee on Prevention, Detection, Evaluation, and Treatment of High Blood Pressure: the JNCL 7 report. JAMA, 289:256-272.

13. Chukwu O (2011). Heart disease, stroke cost Nigerian $\$ 800 \mathrm{~m}$ yearly. Pmnews Sept. 21. Available at http://www.pmnews nigeria.com/2011/09/21/heart-diseasestroke-cost-nigeria-800m yearly 
14. Cooper R, Rotimi C, Ataman S, McGee D, Ositimehin B, Kadiri S, Muna W, et al (1997). The prevalence of hypertension in seven population of West African Origin. American Journal of Public Health, 87 (2): 160-168.

15. Corvol P et al (1992). Can the genetic factors influence the treatment of systemic hypertension? The case of rennin-angiotensin-aldosterone system. American Journal of Cardiology, 70:140-200.

16. Crowther NJ, Cameron N, Trusler J, Gray IP (1998). Association between poor glucose tolerance and rapid post natal weight gain in seven-year-old children. Diabetologia, 41:1163-11167.

17. Daar AS, Singer PA, Persad DL (2007). Grand challenges in chronic noncommunicable diseases. Native, 450-494-6.

18. Dalstra JA et al (2005). Socio-economic differences in the prevalence of common chronic diseases: an overview of eight European countries. International Journal of Epidemiology, 34:316-326.

19. Darnton-H, Coyne ET, Wahlgvist ML (2001). Assesment of nutritional status. In: Ratnaike R. ed. A practical guide to geriatric practice. Sydney, McGraw-Hill, 424439.

20. Ekwunife OI, Aguwa CN (2011). A Meta analysis of prevalence rate of hypertension in Nigerian populations. Journal of Public Health and Epidemiology, 3(13):604-607.

21. Ellison-Loschmann L,et al (2007). Socio-economic status, asthma and chronic bronchitis in a large community-based study. The European Respiratory Journal, 29:87-90.

22. Facchini FS, Hua N, Abbas F,Reaven GM (2001). Insulin resistance as a predictor of age related diseases. J ClinEndocrinol. 86(8):3574-3578.

23. Falck B, Arnio P (1983). Left sided carpel tunnel syndrome in butchers. Scand J Work Environ Health, 9(3): 291-297.

24. Fink RI, Kolterman OG, Griffin J, Olefsky JM (1983). Mechanisms of insulin resistance in aging. J Clin Invest, 71:1523-1535.

25. Folsom AR, Kushi LH, Anderson KE et al (2000). Association of general and abdominal obesity with multiple health outcomes in older women; the lower women's health study. Arch Intern Med., 160:217-228.

26. Fontaine KR, Redden DT, Wang C, Westfall AO, Allison DB (2003). Years of life lost due to obesity. Journal of the American Medical Association, 289:187-193.

27. Godfrey KM, Barker DJ (2000). Fetal nutrition and adult disease. Am J Clin Nutr., 71:1344S-1352S.

28. Grant JE, Hicks N, Taylor AW, Chittleborough CR, Philips PJ (2009).North West Adelaide Health Study Team. Gender-specific epidemiology of diabetes: a representative cross-sectional study. Int J Equity Health, 8:9. Available from http://www.equityhealthj.com/content/8/1/6.

29. Gryglewska B, Grodzicki T, Kocemba J (1998). Obesity and blood pressure in the elderly free-living population. J Hum Hypertens., 12; 645-647. 
30. Healthy Caribbean Coalition (HCC) NCD Alliance-2011. Non-communicable disease poverty for women's health and development. Available from http://www.iumsp.ch/Enseignement/colloques/do.

31. Health Reform Foundation of Nigeria (HERFON) (2011). Diabetes to top cause of death in 2015. Available at http://www.vanguardngr.com/2011/10/diabetes-to-top-cause-of-deathin-2015-healthgroup/.

32. International Diabetes Federation (IDF) $5^{\text {th }}$ Edition of the diabetes Atlas, 2011. Availablefrom http://www.idf.org/diabetesatlas/diabetesandimpairedglucosetolerance.

33. James W, et al (2003). In comparative quantification of health risk: global and regional burden of disease attributable to selected major risk factors. Geneva, World health Organization.

34. Jovanovic-PL, Biermann J. (1996). The diabetic women. New York: GP Putnam's Sons, 16-18.

35. Khatib O (2004). Non-communicable disease: risk factor and regional strategies for prevention and care. Eastern Mediterranean Health Journal, 10(6):77-78.

36. Khuwaja AK, Kadir MM (2010). Gender differences and clustering pattern of behavioral risk factors for chronic non-communicable disease: community-based study from a developing country. Chronic 111 6(3):163-170.

37. Kruger HS, Venter CS, Vorster H, and Margetts BM (2002). Physical inactivity is the major determinant of obesity in black women in the North West Province, South Africa: The THUSA Study. Nutrition, 18:422-427.

38. Levitt NS, Lambert EV, Woods D, Hales CN, Andrews R and Seekl JR (1999). Impaired glucose tolerance and elevated blood pressure and birth weight among 5years- old children from Soweto, South Africa (Birth-to-ten study). Journal of Epidemiology and Community Health 53:264-268.

39. Lopez A, Mathers C, Ezzati M, Jamison D, Murray C (2006). Global Burden of Disease and Risk factors New York, Oxford University Press.

40. Mathew CD, Loncer D (2006). Projection of global mortality and burden of disease from 2002-2030. PLoS Medicine, 3:e442.

41. Meisinger C, Döring A, Thronad B, Heier M, Löwel H(2006). Body fat distribution and risk of type 2 diabetes in the general population: are there differences between men and women? The MONICA/KORA Augsburg Cohort study. Am J Clin Nutr, 84:483-489.

42. Milne EJ, Veriava Y, James SH and Isaacson C (1989). Aetiology and pathogenesis of Malignant Hypertension in black South Africans a Review. South African Medical Journal, 76 (Suppl): 22-23.

43. Misra A and Khurana L (2008). Obesity and the metabolic syndrome in developing countries. J Clin Endocrinol Metab, 93 (11): S9-S30.

44. Mizkurka M, Haddad S, Langlois EV, Freeman E, Kouanada S and Zunzunegui MV (2012). Heavy burden of non-communicable diseases at early age and gender disparities in an adult population of Burkina Faso: World health survey. BMC Health, 12:24.

45. Mohammed OA (2003). Obesity among Saudi male adolescents in Riyadh, Saudi Arabia. Saudi Med J., 24 (1): 27-33. 
46. MOHSS (1997). Federal Ministry of Health and Social Services, Nigeria: The National Expert committee on NCD. Non-communicable diseases in Nigeria. Final Report of National Survey.

47. Mokdad AH, Serdula MK, Dietz WH, Bowman BA, Marks JS, Koplan JP (1999). The spread of the obesity epidemic in the United State 1991 - 1998. J. Am Med. Assoc., 282: 1519-1522.

48. Mo-suwan L, Geater AF (1996). Risk factors for childhood obesity in a transitional society in Thailand. International Journal of Obesity, 20:697-703.

49. Msyamboza KP, Ngwira B, Dzowela T, Mvula C, Kathyola D, Harries AD, Bowle C (2011). The burden of selected chronic non-communicable diseases and their risk factors in Malawi: National STEPS Survey. PLoS One, 6(5):e20316.doi:101371/journal.pone.0020316.

50. Mufunda J, Chatora R, Ndambakuwa Y, Nyarango P, Kosia A., Chifamba J, Filipe A (2006). Emerging Non-communicable disease epidemic in Africa: preventive measures from the WHO Regional office for Africa. Ethnicity and Disease, 16:521-526.

51. Murray C, Lopez A, eds (1996). The global burden of disease. Boston MA, Harvard University Press on behalf of WHO and World Bank.

52. Murray CJL, Lopez AD (1997). Mortality by cause for eight regions of the world: global burden of disease study. Lancet, 340:1269-1276.

53. National Institute of Health (NIH), National Heart, Lung and Blood Institute (1998). Clinical guidelines on the identification, evaluation and treatment of overweight and obesity in adults. The Evidence Report. Obes Res., 6:351-360.

54. Nwachukwu DC, Nwagha UI, Obikili EN, Ejezie FE, Okwuosa CN, Nweke ML (2010). Assessment of blood mass index and blood pressure among university students in Enugu South East, Nigeria. Niger Med., 2:148-152.

55. Peters AJ, Barehderg LJ, Wilekens F, Mackenbach P, Mamum A, Booneux L for NEDCOM (Netherlands Epidemiology and Demography Compression of Morbidity Research Group) (2003).Obesity in adulthood and its consequences for life expectancy: A life table analysis. Annals of Internal Medicine, 1338:24-32.

56. Pihas-Hamiel O, Zeitler PJ (2005). Pediatr, 146. 693-700.

57. Prasanna K (1996). Gender difference in diabetes. Int J Diab Dev Countries., 16:103-104.

58. Rowe JW, Minaker KL, Pallota JA (1983). Characterization of the insulin resistance of aging. J Clin Invest 71:1581-1587.

59. Seedat YK (1999). Hypertension in black South Africans. Journal of Human Hypertension 1999; 13 (2):96-103.

60. Sharma M, Ganguly NK (2005). Premature coronary artery disease in Indians and its associated risk factor. Vasc Health Risk Manag, 1 (3): 217-225.

61. Shukla S (2011). Eat healthy to reduce the risk of non-communicable disease. Asian Tribune 11:282.

62. Stamler JA (2005). Epidemiological findings on BMI and blood pressure in Adults. Ann Epidemiol. 1:347-362. 
63. Tehard B, Friedenreich CM. Oppert JM and Chapelon FC. Effect of physical activity on women at increased risk of breast cancer: Results from the E3N Cohort Study, cancer Epidemiol Biomarkers Prev 2006; 15:57-64.

64. Thankappan KR, Shah B, Mathur P, Sarma PS, Srinivas G, Mini GK, Daivdanam $\mathrm{M}$ et al(2010). Risk factors profile for chronic non-communicable diseases: Results of a community- based study in Kerala, India. Indian J Med Res, 131:53-63.

65. Thiam I, Samba K, Lwanga D (2006). Diet-related chronic disease and double burden of malnutrition in West Africa. United Nation Standing Committee on Nutrition News, 33.

66. Unwin N, Setel P, Rashid S, Mugusi F, Mbanya JC, Kitange H, Hayes L, Edwards R, Aspray T, Alberti KG (2001). Non-communicable diseases in sub-Saharan Africa: where do they feature in the health research agenda? Bulletin of World Health Organization, 79(10):947-953.

67. Vander Sande MA, Walraven GE, Milligan PJ, Banya WA, Ceesay SM, Nyan OA, Mcdam PW. Family history: an opportunity for early interventions and improved control of hypertension obesity and diabetes. Bulletin of the World health organization 2001; 79 (4):321-328.

68. Venkat Narayan KM, Ali MK, Koplan JP (2010). Global non-communicable disease-where worlds meet. New England Journal of Medicine, 363(13):1196-1198.

69. WHO (2000).Obesity: Preventing and managing the global epidemic: environmental and societal influences, World Health Organization, 894:118-122.

70. WHO (2003). Screening for Type 2 diabetes. Report of a WHO and IDF Meeting, Geneva, World Health Organization.

71. WHO (2004).Comparative quantification of health risks: Global and regional burden of disease attributable to selected major risk factors. Geneva, World Health Organization.

72. WHO (2005). Preventing chronic diseases, a vital investment. Geneva, World Health Organization.

73. WHO (2008). Waist circumference and waist-hip ratio. A report of a WHO Expert Consultation, Geneva, World Health Organization.

74. WHO (2008). The global burden of disease: 2004 update, Geneva, World Health Organization.

75. WHO (2009).Global Health Risk; mortality and burden of disease attributable to selected major risk, Geneva: World Health Organization.Available: http://www.who.int/healthinfo/global_burden_disease/globalhealthriske_report_full.pdf.

76. WHO (2010). World Health Organization (WHO) Global Status Report on noncommunicable disease. Available at: http://www.who.int.nmh/publications/ncd report full enpdf.

77. WHO (2011). WHO Maps: Non-communicable disease trend in all countries, World Health Global Report, World Health Organization.

78. Wild S, Roglic G, Green A, Sicree R, King H (2004). Global prevalence of diabetes estimates for the year 2000 and projection to 2030. Diabetes Care, 27:1047-153.

79. Ziraba AK, Fotso JC, Ochako R (2009). Overweight, obesity and urban Africa: A problem of the rich or the poor? BMC Public Health, 9:465. 